\title{
Time-Independent Schrödinger Equation as a Straight Line Equation in Cartesian Coordinates
}

\author{
Wojciech Szczepankiewicz
}

\begin{abstract}
A graphical interpretation of the time-independent Schrödinger equation in the Cartesian coordinate system for the explicitly set wave function of the ground state and selected excited states of the hydrogen has been presented. It was shown that the equation left-sidedly multiplied by the function $\psi$ allows to find its didactic connection with the course of the beam of light emitted by the hydrogen atom in the measuring system of the spectroscope equipped with a prism.
\end{abstract}

Index Terms - Cartesian coordinates, prism, Schrödinger equation, straight line.

\section{INTRODUCTION}

The inspiration to write the present paper was the remark made in the book [1], which is as follows: "We are so used to simple proportionality that we are liable to underrate the far-reaching consequences of this simple law.". Although this sentence refers to some phenomena occurring in the cells of living organisms, we will show below that it also applies to the time-independent Schrödinger equation (hereinafter abbreviated as TISE). Proportionality in the considered equation is limited only to special examples of the so-called own functions, yet these are fundamental examples. We will also demonstrate that the proportionality analysis allows to find a connection between a TISE geometric image and a spectroscopic experiment in which a beam of light from an atom that emits radiation is split into components by means of a prism.

Until now, the TISE description in teaching literature has focused on numerical solutions of one-dimensional TISE [2],[3]. Calculation of adiabatic approximation of TISE solutions [ 4 ], application of Mathematica computer application to obtain some solutions of TISE [5],[6]. WKB approximation in the TISE solution [7]. PIB (particle in box) solution [8], the influence of the configuration interaction (CA) method on the TISE solutions [9]. TISE solutions for two-atom oscillators using linear algebra methods [10]. TISE solutions with the use of anharmonic potentials [11]. The graphic side of TISE was discussed in relation to the connection of angular momentum of an electron and other quantum numbers [12].

\section{THE SCHRÖDINGER EQUATION IN THE PARAMETRIC CARTESIAN COORDINATE SYSTEM}

The time independent Schrödinger equation is recorded in the commonly known formula (1):

Wojciech Szczepankiewicz, Faculty of Chemistry, Silesian University of Technology, Gliwice, Poland
$\hat{H} \psi=E \psi$

The external form of (1) may suggest that it is an advanced analogue of the ordinary straight line equation $y=a \cdot x$, which is an equation with $a$ angular coefficient of a straight line passing through the origin of the Cartesian coordinate system. However, in the case of (1) we can understand that all the values $\psi$ should be set aside on the $x$-axis, while $\hat{H} \psi$ ones should be set on the $y$-axis, as far as both the Hamiltonian and function $\psi$ are given explicitly. With this assumption, we would deal with a parametric coordinate system, where the parameters would be all the independent variables of $\psi$ and possible constant parameters on which the function $\psi$ could depend. In such a system, the value $\mathrm{E}$ would act as the angular coefficient of a certain straight line, which should also pass through the origin of this system. We will check if this assumption for a few selected forms of (1).

Let us consider a hydrogen atom with the Born-Oppenheimer approximation and with the infinitely heavy nucleus. In this arrangement, the Hamilton operator for the hydrogen atom takes the form of (2) in atomic units:

$$
\hat{H}=-\frac{1}{2}\left(\frac{\delta^{2}}{\delta x^{2}}+\frac{\delta^{2}}{\delta y^{2}}+\frac{\delta^{2}}{\delta^{2}}\right)-\frac{1}{r}
$$

Let us arbitrarily assume the form of a wave function $\psi$, which is a Q-class function and may be intended to describe some state of a hydrogen atom. Let this function be $\psi=e^{-c r}$ [13], where $c$ is a certain non-negative constant (so called a vartiational constant), while $r$ is the radius vector of the electron. Additionally, the proton is placed at the origin of the coordinate system. After these assumptions, TISE will transform to the following form (3):

$$
\hat{H}=-\frac{1}{2}\left(\frac{\delta^{2}}{\delta x^{2}}+\frac{\delta^{2}}{\delta y^{2}}+\frac{\delta^{2}}{\delta^{2}}\right) e^{-c r}-\frac{1}{r} e^{-c r}=E e^{-c r}
$$

Drawing a line course in a parametric coordinate system will be possible after carrying out the Hamiltonian operation on the selected function. For this purpose, the sum of the second derivatives of the selected function $\psi$ have to be calculated, remembering that the radius vector is in the following form: $r=\sqrt{x^{2}+y^{2}+z^{2}}$. It is convenient to carry out the differentiation procedure using one of the symbolic or on-line calculation algorithms available on websites designed for such calculations. After carrying out the differentiation and ordering of variables, e.g. such a form of TISE is obtained (4): 
$\left(-\frac{1}{2} c^{2}-\frac{c-1}{r}\right) e^{-c r}=E e^{-c r}$

It is obvious that we are now able to divide both sides of (4) by $e^{-c r}$ and obtain the expression (5) for energy as a function of two variables, $c$ and $r$ :

$$
E=-\frac{1}{2}+\frac{c-1}{r}
$$

It should be noted that only if $c=1$, the energy value is a constant one and it is equal to $\mathrm{E}=-0.5$. In fact, it is the energy of the ground state of the hydrogen atom, expressed in the atomic unit (hartree). For this value of $c$ the energy of the system is constant and all the possible electron positions in the space surrounding the nucleus.

However, our aim is to present (4) in the Cartesian coordinate system. This can be done by selecting one of the available websites with online procedures to draw diagrams of parametric equations and apply (4) directly for the selected value $c$ with the parameter $r$. Instead of this, we used Visual Basic programming language [14] (see Appendix, Procedure 1) for this purpose. Fig. 1 shows the eq. (4) curves plotted for three arbitrarily chosen values of $c(0.2,1.0$ and 1.8$)$ with $r$ used as a parameter within range from 0.01 to 15 :

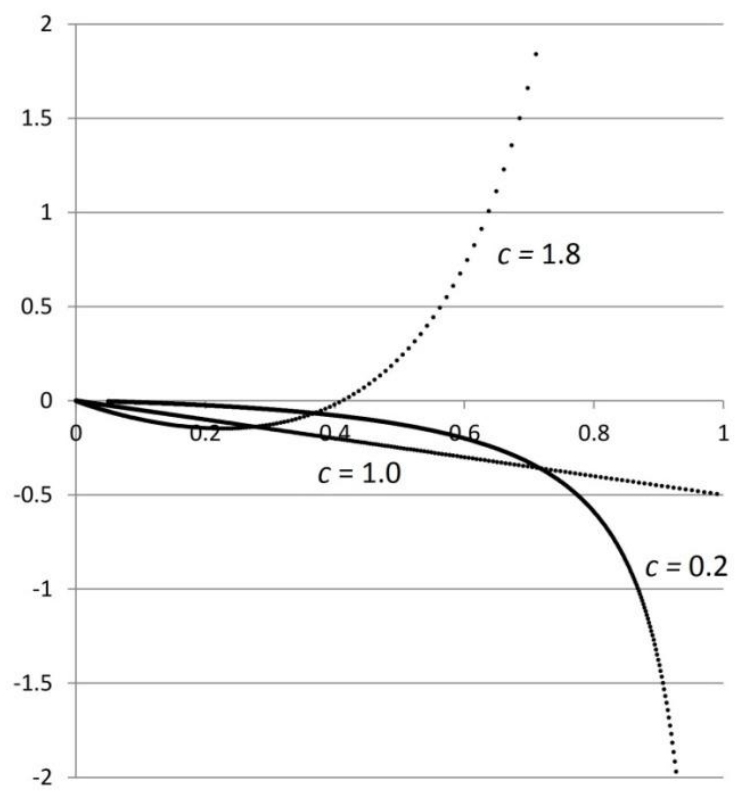

Fig. 1. MS Excel chart showing eq. (5) plots for three different values of the coefficient $c$.

In reality, a straight line is formed in the system only for values $c=1$. For other values, certain curves are formed. This always happens when the function $\psi$ it is not an eigenfunction of the Hamiltonian (obviously when $c \neq 1$ in this case).

We will consider one more example of the function $\psi$, which becomes negative for some values of the radius vector $r$ and additionally some values of this function depend on the angles in the spherical coordinate system. Let this be a function (6):

$\psi=2 c r \sin (\theta) \cos (\phi) e^{-c r}$ which is an eigenfunction corresponding to the unnormalized orbital $2 p_{x}$ only when $c=0.5$. The Hamiltonian operation on this function gives (7):

$$
\hat{H} \psi=-2 c\left(\frac{1}{2} c(c r-4)+1\right) \sin (\theta) \cos (\phi) e^{-c r}
$$

Using (7), the full version of the time-independent Schödinger equation takes the form of (8):

$-2 c\left(\frac{1}{2} c(c r-4)+1\right) \sin (\theta) \cos (\phi) e^{-c r}=E 2 c r \sin (\theta) \cos (\phi) e^{-c r}$

As (8) is dependent not only on $r$ and $c$ but additionally on the angles $\theta$ and $\phi$, this fact should be taken into account when constructing a program for drawing a straight line. This means the need to introduce additional two loops to change the characteristic angles across their function domain (see appendix).

The function $2 p_{x}$ has negative values for a certain array $r$ $\theta$ and $\phi$. This fact is manifested by the appearance of the straight line arm in the second quadrant of the parametric coordinate system (Fig. 2, Appendix, Procedure 2):

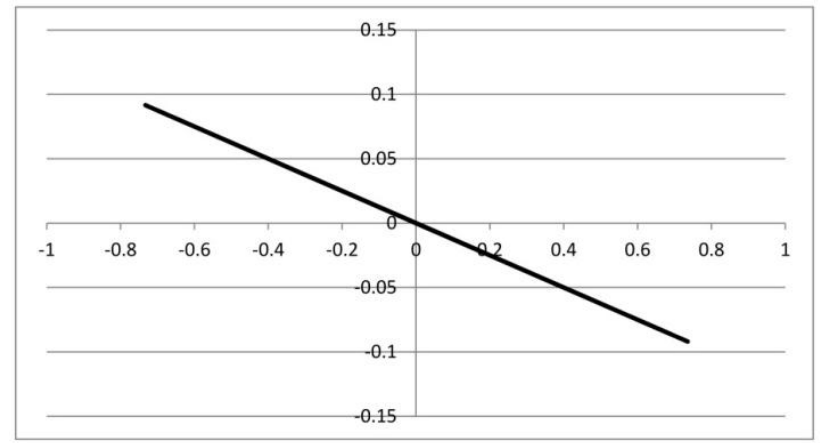

Figure 2. An Excel chart showing eq. (8) for $c=0.5$.

Such simple figures can be made for each eigenfunction of the selected quantum state of the hydrogen atom. For training purposes, the results of the Hamiltonian operation on functions $2 s, 2 p_{y}, 2 p_{z}$ and all functions (unnormalized orbitals) of the $\mathrm{M}$ shell are given in the appendix. They can be used for the exercise of examining for which values $c$ a straight line is formed in the coordinate system $(\psi, \hat{H} \psi)$.

It should be noted that in a three-dimensional Cartesian coordinate system, e.g. $(r, \psi, \hat{H} \psi)$, the presented straight lines are only projections of spatial curves on the plane $\psi, \hat{H} \psi$. These curves have asymptotes on the axis $r$ for $r \rightarrow \infty$ regardless of the parameter value $c$.

\section{CONNECTION OF THE PARAMETRIC COORDINATE} SYSTEM WITH THE REAL SPECTROSCOPIC EXPERIMENT

The element relevant for didactics of quantum chemistry appears only when TISE is left-sidedly multiplied by the function $\psi(9)$ :

$$
\psi \hat{H} \psi=E \psi^{2}(9)
$$

We will use this last form to create a Cartesian coordinates with the axes defined as $\left(\psi^{2}, \psi \hat{H} \psi\right)$. It turns out that this 
procedure causes that straight line points are located only in the 4th quadrant of the coordinate system for both positive and negative values for an eigenfunction of the adopted Hamiltonian (Fig. 3, Appendix, Procedure 3):

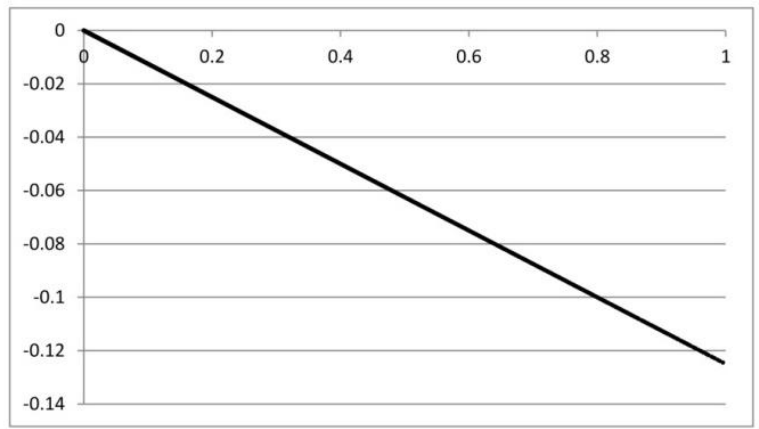

Figure 3. The course of a straight line in a coordinate system $\left(\psi^{2}, \psi \hat{H} \psi\right)$ for $\psi=2 p_{x}$.

This property causes that we can identify the behaviour of such a straight line with the geometrical system of a prism used e.g. in a UV/Vis spectrophotometer in which takes place the diffraction of radiation emitted, e.g. by the hydrogen atom. Let us suppose we throw a collimated light beam corresponding, e.g. to the Balmer series boundary, whose energy is equal to $-3.40 \mathrm{eV}$ ( $410 \mathrm{~nm}$ or -0.12495 hartree). Let us additionally suppose that this beam falls on the prism at the angle $\beta$ selected by us in such a way that the tangent of the deflection angle $\alpha$ is numerically equal to the energy in the selected physical unit. This situation is presented in Fig. 4:

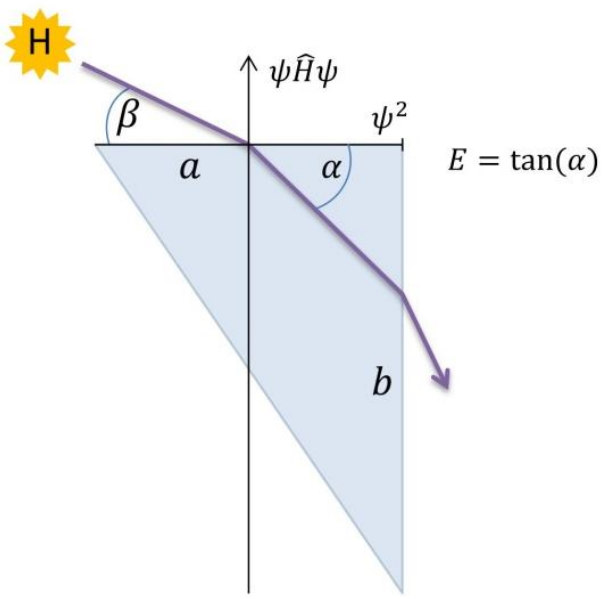

Fig. 4. A prism with a beam of light course emitted by a $\mathrm{H}$ source in the coordinate system $\left(\psi^{2}, \psi \hat{H} \psi\right)$.

In such a system, we can assign the edge $a$ of the prism with the role of the axis $\psi^{2}$, which adopts the maximum value (i.e. for $\psi^{2}=0$ ) at the point of contact with the perpendicular edge $b$ and at the same time where the light beam leaves the prism. On the other hand, the vertical edge $\psi \hat{H} \psi$ is routed perpendicular to the axis $\psi^{2}$. The origin of the coordinate system is placed at the point where the collimated light beam falls into the prism. It can be seen from the Fig. 3 that such a connection concerns the course of the beam of light only inside the prism. The angular coefficient of such a line still corresponds to the energy of the selected quantum state of the hydrogen atom. It can be seen that the selection of any trial function which is not the own function of the chosen Hamiltonian (e.g. by changing the value c) causes the system to obtain a curve that has an asymptote for the maximum value $\psi^{\wedge} 2$. It can be said that the trial functions, which are not eigenfunctions, give such a course of the beam of light inside the prism that it does not come out in the expected place. This means that trial functions that are not self-functions are nonphysical in the sense that they in a prism, they behave in a rather unusual way for a light beam, and that obtaining solid values (independent of the radius vector and angles) of quantum energy values requires additional mathematical operations, e.g. the averaging process involving the integration of (8) in the entire electron position variability area.

It should be noted that more than one radius from excited hydrogen atom states can be placed in Figure 4. To make this possible, the functions $\psi$ should be normalised in such a way that their maximum values are the same (the normalisation procedure was carried out in the case of Figure 3 - see supplement). This is not a normalisation characteristic for orbitals. There it is required that the integral of the square of the wave function is equal to one. Here, it is enough for the maximum value of all selected functions to be e.g. 1 .

\section{APPENDIX}

Main symbols:

$a$ - coefficient at the Laplacian in atomic units

$b$-coefficient at the potential in atomic units

$c$ - variational variable

$r$ - electron radius vector

$p s i-\psi$, wave function variable

$H p s i-\hat{H} \psi$, function as the result of the Hamiltonian action on the function $\psi$.

All the procedures are written in MS Visual Basic for Excel.

Procedure 1.

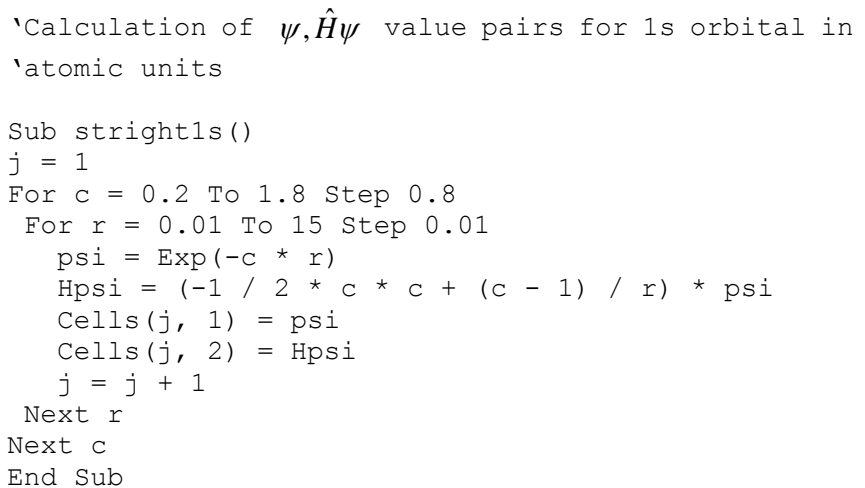

Procedure 2.

'Calculation of $\psi, \widehat{H} \psi$ value pairs for 'unnormalized $2 \mathrm{px}$ orbital in atomic units 


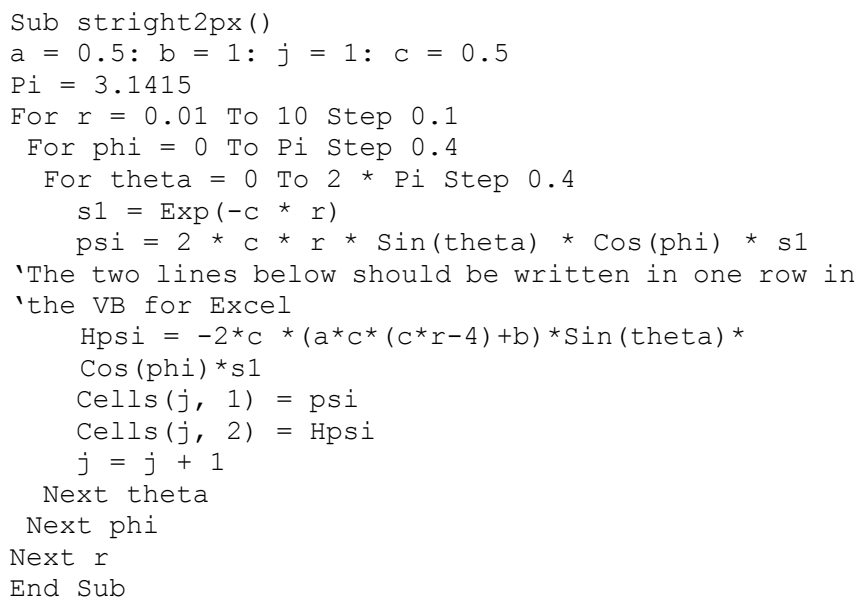

[13] C. A. Coulson, Valences. London: Oxford University Press, 1961. [14] MS Excel is owned by Microsoft corp.

Dr. Wojciech Szczepankiewicz, DSc., organic chemists at he Department of Organic Chemistry, Bioorganic Chemistry, Faculty of Chemistry, Silesian University of Technology, Gliwice, Upper Silesia, Poland.

Procedure 3.

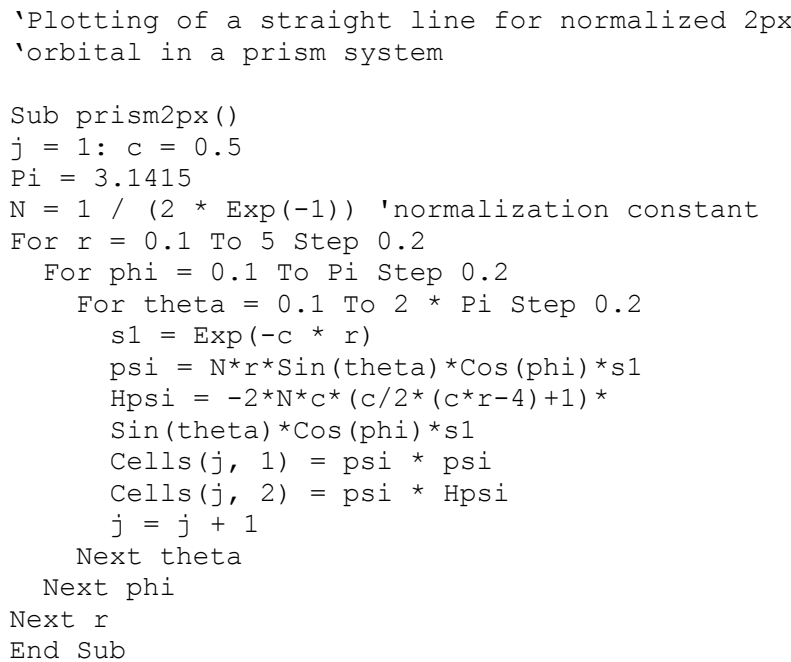

\section{REFERENCES}

[1] E. Schrödinger, What is life? The Physical Aspect of the Living Cell. Cambridge: Cambridge University Press, 1948.

[2] U. Blukis, J. M. Howell, "Numerical solution of the one-dimensional Schrödinger equation", J. Chem. Edu., 1983, 60, 207-212.

[3] J. Tellinghuisen, J. "Accurate Numerical Solutions of the One-Dimensional Schrodinger Equation”, J. Chem. Edu.,1989, 66, 51.

[4] A. Daniel, D. A. Morales, "On the use of the adiabaticity concept to obtain approximate solutions of the Schrödinger equation", J. Chem. Edu.,1990, 67, 211-213.

[5] J. C. Hansen, "Schroedinger.m: A Mathematica Package for Solving the Time-Independant Schrödinger Equation", J. Chem. Edu.,1996, 73, 523.

[6] J. C. Hansen, "Schroedinger.m: A Mathematica Package for Solving the Time-Independent Schrödinger Equation". J. Chem. Edu., 1996, 73, 924

[7] Z. L. Gasyna, J. C. Light, "Application of the WKB Approximation in the Solution of the Schrödinger Equation", J. Chem. Edu., 2002, 79, 133-134.

[8] P. F. Newhouse, P. F.; K. C. McGill, "Schrödinger Equation Solutions That Lead to the Solution for the Hydrogen Atom", J. Chem. Edu.,2004, $81,424-426$.

[9] J. A. Sordo, "The Configuration Interaction Expansion Is the Exact Solution of the Electronic Schrödinger Equation", J. Chem. Edu., 2006, $83,480$.

[10] Z. L. Gasyna, ,Solution of the Schrödinger Equation for a Diatomic Oscillator Using Linear Algebra", J. Chem. Edu.,2008, 85, 845-846.

[11] G. S. Beddard, "Solution of the Schrödinger Equation for One-Dimensional Anharmonic Potentials: An Undergraduate Computational Experiment”, J. Chem. Edu., 2011, 88, 929-931.

[12] T. A. Francis, D. G. Miles Jr. "A Graphical Approach to the Angular Momentum Schrödinger Equation” J. Chem. Edu., 78, 2001, 405-408. 